PROCEEDINGS OF THE AMERICAN MATHEMATICAL SOCIETY

Volume 124, Number 8, August 1996

\title{
NONRESONANCE PROBLEMS FOR DIFFERENTIAL INCLUSIONS IN SEPARABLE BANACH SPACES
}

\author{
ZOUHUA DING AND ATHANASSIOS G. KARTSATOS
}

(Communicated by Hal L. Smith)

\begin{abstract}
Let $X$ be a real separable Banach space. The boundary value problem

$$
\begin{aligned}
& x^{\prime} \in A(t) x+F(t, x), t \in \mathcal{R}_{+}, \\
& U x=a,
\end{aligned}
$$

is studied on the infinite interval $R_{+}=[0, \infty)$. Here, the closed and densely defined linear operator $A(t): X \supset D(A) \rightarrow X, t \in \mathcal{R}_{+}$, generates an evolution operator $W(t, s)$. The function $F: \mathcal{R}_{+} \times X \rightarrow 2^{X}$ is measurable in its first variable, upper semicontinuous in its second and has weakly compact and convex values. Either $F$ is bounded and $W(t, s)$ is compact for $t>s$, or $F$ is compact and $W(t, s)$ is equicontinuous. The mapping $U: C_{b}\left(\mathcal{R}_{+}, X\right) \rightarrow X$ is a bounded linear operator and $a \in X$ is fixed. The nonresonance problem is solved by using Ma's fixed point theorem along with a recent result of Przeradzki which characterizes the compact sets in $C_{b}\left(\mathcal{R}_{+}, X\right)$.
\end{abstract}

\section{Introduction. Preliminaries}

Throughout the paper, $X$ is a real, separable Banach space with dual space $X^{*}$. We also denote by $L(X)$ the space of bounded linear operators $V: X \rightarrow X$. The symbols $\mathcal{R}, \mathcal{R}_{+}$and $\mathcal{R}^{+}$stand for the sets $(-\infty, \infty),[0, \infty)$ and $(0, \infty)$, respectively. We consider differential inclusions of the type

$$
x^{\prime} \in A(t) x+F(t, x), x(0)=x_{0},
$$

where, for $t \in \mathcal{R}_{+}$, the linear operator $A(t): X \supset D(A) \rightarrow X$ is the generator of a compact evolution operator $W(t, s) \in L(X), 0 \leq s \leq t<+\infty$, and $F: \mathcal{R}_{+} \times X \rightarrow$ $X$ is a set-valued mapping. We always assume that $A(t)$ is closed and densely defined on its domain $D(A) \equiv D(A(0))$, which is independent of $t$. In Section 2 we obtain the solvability of the associated boundary value problem

$$
\begin{aligned}
& x^{\prime} \in A(t) x+F(t, x), t \in \mathcal{R}_{+}, \\
& U x=a,
\end{aligned}
$$

where $U: C_{b} \rightarrow X$ is a bounded linear operator and $a \in X$ is fixed. Here, $C_{b} \equiv C_{b}\left(\mathcal{R}_{+}, X\right)$ is the Banach space of all bounded and continuous functions

Received by the editors December 16, 1994.

1991 Mathematics Subject Classification. Primary 34A60.

Key words and phrases. Boundary value problem on an infinite interval, differential inclusion, upper semicontinuous function, compact evolution operator.

(C)1996 American Mathematical Society 
$f: \mathcal{R}_{+} \rightarrow X$ associated with the sup-norm. The "single-valued" problem (B) was studied in finite-dimensional cases by Kartsatos in [6] and [8]. The EilenbergMontgomery fixed point theorem on finite intervals was used by Kartsatos [7]. These results were extended by Anichini [1] to more general quasilinear cases. A boundary value problem for closed and densely defined principal parts was considered by Kartsatos [8], but the boundary conditions there could not include the periodic ones. A problem involving boundary conditions on finite intervals, like the ones used in this paper, was studied by Ward [17]. In 1979, Zecca and Zezza [18] considered the case of an infinite interval and differential inclusions in Banach spaces, but for bounded linear operators in the principal parts of the corresponding inclusions. Unbounded linear operators for differential inclusions were considered by Papageorgiou [14] and $\mathrm{Hu}$ and Papageorgiou [5]. It seems that the problem considered here has not been attacked, in this generality, by any other author. Further references on these problems can be found in the above cited papers and the references therein.

We let

$$
\mathcal{P}_{f(c)}(X) \equiv\{A \subset X: A \text { is nonempty, closed (and convex) }\} .
$$

We also let

$$
\mathcal{P}_{w k c}(X) \equiv\{A \subset X: A \text { is nonempty, convex and weakly compact }\} \text {. }
$$

We denote by $\mathcal{N}$ the set of all positive integers. We also denote by $\Delta$ the set $\{(t, s): 0 \leq s \leq t \leq T\}$. The symbol $B_{r}(0)$ stands for the open ball of $X$ with radius $r>0$. We denote by $\operatorname{co} A$ (resp. $\overline{\mathrm{co}} A$ ) the convex (resp. closed convex) hull of the set $A$. For an interval $J \subset \mathcal{R}_{+}$, the symbol $L_{+}^{1}(J, \mathcal{R})$ stands for the set of all nonnegative functions in $L^{1}(J, \mathcal{R})$.

Definition 1. Let $J$ be an interval of $\mathcal{R}_{+}$. Let $H: J \rightarrow \mathcal{P}_{f}(X)$. Then $H$ is called "measurable" if for every open set $D \subset X$ the set $\{t \in J: H(t) \bigcap D \neq \emptyset\}$ is measurable. For such a measurable function $H$, and for $J=(0, T), T \in(0, \infty]$, we set

$$
S_{H}^{1}(T) \equiv\left\{f \in L^{1}(J, X) \text { such that } f(t) \in H(t) \text { a.e. in } J\right\} .
$$

A function $H: J \rightarrow 2^{X}$ is called "integrably bounded" if there exists $m \in L_{+}^{1}(J, R)$ such that, for almost all $t \in J$, we have $H(t) \subset m(t) \overline{B_{1}(0)}$.

The following result can be found in Papageorgiou [13, Theorem 3.1].

Lemma 1. If $H: J \rightarrow \mathcal{P}_{w k c}(X)$ is integrably bounded, where $J$ is an interval of $\mathcal{R}_{+}$with endpoints $0, T(T \leq \infty)$, then the set $\mathcal{S}_{H}^{1}(T)$ is a nonempty, weakly compact and convex subset of $L^{1}((0, T), X)$.

For various measurability results concerning multi-valued functions, the reader is referred to Himmelberg [4]. We also follow Papageorgiou [14] for facts involving the measurability/integrability of the various mappings considered in this paper.

The evolution operators $W(t, s)$ considered in this paper (cf. Friedman [3]) satisfy the following conditions.

(j1) $W(t, s) \in L(X)$ for every $(t, s) \in \Delta$ and for each $x \in X$ the mapping $(t, s) \rightarrow$ $W(t, s) x$ is continuous;

(j2) $W(t, s) W(s, r)=W(t, r)$ whenever $0 \leq r \leq s \leq t \leq T$;

(j3) $W(t, t)=I$, i.e., the identity operator on $X$ for all $t \in[0, T]$; 
(j4) the mapping $(s, T] \ni t \rightarrow W(t, s) \in L(X)$ is continuous with respect to the uniform operator topology of $L(X)$. Moreover, this continuity is uniform with respect to $s$ lying in sets bounded away from $t$, i.e., as long as $t-s \geq \beta$ for any fixed $\beta>0$.

We observe that an evolution operator $W(t, s)$ satisfying the above conditions (j1)(j3) is uniformly bounded, i.e., there exists a constant $p(W)>0$ such that

$$
\|W(t, s)\| \leq p(W),(t, s) \in \Delta .
$$

This follows easily from the Uniform Boundedness Principle.

The following lemma is an important tool in this paper. Its proof is given for the sake of completeness and future reference.

Lemma 2. Let $\left\{f_{\alpha}(t)\right\}_{\alpha \in \mathcal{A}}$ be a set of $L^{1}((0, T), X)$-functions such that

$$
\left\|f_{\alpha}(t)\right\| \leq f(t) \text {, for almost all } t \in[0, T] \text { and all } \alpha \in \mathcal{A},
$$

where $f \in L_{+}^{1}((0, T), \mathcal{R})$. Assume that conditions $(\mathrm{j} 1)-(\mathrm{j} 4)$ are true for the evolution operator $W(t, s)$. Then the set

$$
M_{1} \equiv\left\{u \in C([0, T], X): u(t)=\int_{0}^{t} W(t, s) f_{\alpha}(s) d s, \text { for some } \alpha \in \mathcal{A}\right\}
$$

is equicontinuous on the interval $[0, T]$.

Proof. From Theorem 6.5 of Himmelberg [4], we know that $s \rightarrow W(t, s) f_{\alpha}(s)$ is measurable. We also know that this mapping is integrably bounded over the interval $[0, t]$. Obviously, we may assume that the function $f(t)$ has a positive integral over the interval $[0, T]$. It suffices to show that $M_{1}$ is equicontinuous at each $t_{0} \in$ $[0, T]$. Fix $t_{0} \in(0, T)$ and let $\epsilon>0$ and $\lambda \in\left(0, t_{0}\right)$ be such that $t_{0}+\lambda<T$ and

$$
p(W) \int_{\Omega} f(s) d s<\epsilon / 4,
$$

where $\Omega$ is any subset of $[0, T]$ with measure $m(\Omega) \leq 2 \lambda$. Now, let $\delta=\delta\left(t_{0}, \lambda, \epsilon\right)<\lambda$ be such that

$$
\left\|W\left(t_{0}+h, s\right)-W\left(t_{0}, s\right)\right\| \leq\left[\epsilon /\left(2 \int_{0}^{T} f(s) d s\right)\right], s \in\left[0, t_{0}-\lambda\right],|h| \in[0, \delta] .
$$

This is possible by the uniform continuity of the mapping $[0, T] \ni t \rightarrow W(t, s) \in$ $L(X)$, for $t-s \geq \lambda$. Then, for $u \in M_{1}$ and some $\alpha \in \mathcal{A}$,

$$
\begin{aligned}
u\left(t_{0}+h\right)-u\left(t_{0}\right)= & \int_{0}^{t_{0}+h} W\left(t_{0}+h, s\right) f_{\alpha}(s) d s-\int_{0}^{t_{0}} W\left(t_{0}, s\right) f_{\alpha}(s) d s \\
= & \int_{t_{0}-\lambda}^{t_{0}+h} W\left(t_{0}+h, s\right) f_{\alpha}(s) d s-\int_{t_{0}-\lambda}^{t_{0}} W\left(t_{0}, s\right) f_{\alpha}(s) d s \\
& +\int_{0}^{t_{0}-\lambda}\left[W\left(t_{0}+h, s\right)-W\left(t_{0}, s\right)\right] f_{\alpha}(s) d s .
\end{aligned}
$$

We have

$$
\left\|\int_{t_{0}-\lambda}^{t_{0}+h} W\left(t_{0}+h, s\right) f_{\alpha}(s) d s\right\| \leq p(W) \int_{t_{0}-\lambda}^{t_{0}+h} f(s) d s<\epsilon / 4
$$




$$
\left\|\int_{t_{0}-\lambda}^{t_{0}} W\left(t_{0}, s\right) f_{\alpha}(s) d s\right\| \leq p(W) \int_{t_{0}-\lambda}^{t_{0}} f(s) d s<\epsilon / 4 .
$$

From these estimates and (2), it follows that

$$
\left\|u\left(t_{0}+h\right)-u\left(t_{0}\right)\right\|<\epsilon \text {, for all } h \in \mathcal{R} \text { with }|h| \leq \delta .
$$

Thus, $M_{1}$ is equicontinuous at every $t_{0} \in(0, T)$. The equicontinuity of $M_{1}$ at $t_{0}=0$ follows from

$$
\|u(h)\| \leq p(W) \int_{0}^{h} f(s) d s, \text { for every } h \in[0, T] .
$$

To see the equicontinuity of $M_{1}$ at $t_{0}=T$, it suffices to observe that

$$
\begin{aligned}
u(T)-u(T-h)= & \int_{0}^{T-2 \lambda} W(T, s) f_{\alpha}(s) d s+\int_{T-2 \lambda}^{T} W(T, s) f_{a}(s) d s \\
& -\left(\int_{0}^{T-2 \lambda} W(T-h, s) f_{\alpha}(s) d s+\int_{T-2 \lambda}^{T-h} W(T-h, s) f_{\alpha}(s) d s\right) \\
= & \int_{0}^{T-2 \lambda}[W(T, s)-W(T-h, s)] f_{\alpha}(s) d s \\
& +\int_{T-2 \lambda}^{T} W(T, s) f_{\alpha}(s) d s-\int_{T-2 \lambda}^{T-h} W(T-h, s) f_{\alpha}(s) d s,
\end{aligned}
$$

where we have assumed that: given $\epsilon>0$ we pick $\lambda \in(0, T / 2)$ so that

$$
p(W) \int_{\Omega} f(s) d s<\epsilon / 4,
$$

for every measurable set $\Omega \subset[0, T]$ with $m(\Omega)<2 \lambda$. We have also assumed $\delta=$ $\delta(\epsilon, \lambda, T) \in(0, \lambda)$ is chosen so that

$$
\sup _{s \in[0, T-2 \lambda]}\|W(T, s)-W(T-h, s)\|<\left[\epsilon /\left(2 \int_{0}^{T} f(s) d s\right)\right],
$$

for all $h \in \mathcal{R}_{+}$with $h \in[0, \delta]$. The rest of the proof follows as above. It is therefore omitted.

The function $F(t, x)$ is always assumed to be defined on $\mathcal{R}_{+} \times X$ and such that $F(t, x(t))$ admits a measurable selection for all $x \in C\left(\mathcal{R}^{+}, X\right)$.

Definition 2. A function $x \in C\left(\mathcal{R}_{+}, X\right)$ is called a "mild solution" of (1) if there exists $f \in S_{F(\cdot, x(\cdot))}^{1}(\infty)$ such that

$$
x(t)=W(t, 0) x_{0}+\int_{0}^{t} W(t, s) f(s) d t, t \in \mathcal{R}_{+} .
$$

\section{Boundary VAlue PROBlems on INFINITE INTERVALS}

The following condition will be needed in the sequel.

(i1) The operator $\widetilde{U}$, defined by

$$
\widetilde{U} x \equiv U(W(\cdot, 0) x), x \in X,
$$

has a bounded inverse $\widetilde{U}^{-1}: X \rightarrow X$. 
A mild solution $x \in C\left(\mathcal{R}_{+}, X\right)$ of problem (1) satisfies $U x=a$, under condition (i1), if and only if it satisfies

$$
x(t)=W(t, 0) \widetilde{U}^{-1}[a-U \phi(\cdot, f)]+\int_{0}^{t} W(t, s) f(s) d s, t \in \mathcal{R}_{+},
$$

where

$$
\phi(t, f) \equiv \int_{0}^{t} W(t, s) f(s) d s .
$$

The function $f$ here is an $L^{1}\left(\mathcal{R}^{+}, X\right)$-function such that $f(t) \in F(t, x(t))$ for almost all $t \in \mathcal{R}_{+}$, i.e., $f \in S_{F(\cdot, x(\cdot))}^{1}(\infty)$. Such a function $x(t), t \in \mathcal{R}_{+}$, is called a "mild solution" of problem (B).

Kartsatos introduced in [6] a method for studying such problems in $\mathcal{R}^{n}$. In the present setting, this method is based on "solving" first a certain problem in each Banach space $C_{m}=C_{m}\left(\mathcal{R}_{+}, X\right)$ of truncated functions defined by

$$
C_{m}=\left\{f \in C\left(\mathcal{R}_{+}, X\right): f(t)=f(m) \text { for all } t \in[m, \infty)\right\} .
$$

A sequence $\left\{x_{m}\right\}$ of "solutions", with $x_{m} \in C_{m}$, would then produce the desired mild solution of problem (3). Unfortunately, we have not been able to apply this method to the present setting. We leave it thus as an open problem.

In another vein, one may work directly with the space $C_{b}$. This has been possible, since 1992, because Przeradzki gave in [16] the following chacterization of the compact sets in $C_{b}$.

Theorem A. A set $B \subset C_{b}$ is compact if and only if the following conditions are satisfied:

(i) for every $t \in R_{+}$, the set $\{x(t): x \in B\}$ is relatively compact in $X$;

(ii) for every $\alpha>0$, the set $B$ is equicontinuous on the interval $[0, \alpha]$;

(iii) for every $\epsilon>0$ there exist $T=T(\epsilon)>0$ and $\delta=\delta(\epsilon)>0$ such that if $x, y \in B$ with $\|x(T)-y(T)\| \leq \delta$, then $\|x(t)-y(t)\| \leq \epsilon$ for all $t \in[T, \infty)$.

Przeradzki gave his result for compact subsets $B$ of $C(\mathcal{R}, X)$, but his proof carries over trivially to our case above. Theorem 1 below uses Theorem A in order to solve problem (B). In what follows,

$$
B_{M}^{C}(0) \equiv\left\{f \in C_{b}:\|f\|_{\infty} \leq M\right\},
$$

where $M$ is a fixed positive constant. A function $F: \mathcal{R}_{+} \times X \rightarrow 2^{X}$ is "bounded" if, for every bounded interval $J \subset \mathcal{R}_{+}$and every bounded set $B \subset X$, there exists a bounded set $Q \subset X$ such that $F(t, x) \subset Q$, for almost all $t \in J$ and all $x \in B$. F is "compact" if the bounded set $Q$ is replaced by a compact set $Q$.

Theorem 1. Let $A(t): X \supset D(A) \rightarrow X, t \in \mathcal{R}_{+}$, generate an evolution operator $W(t, s)$ satisfying conditions $(\mathrm{j} 1)-(\mathrm{j} 4)$ on every compact interval $[0, T], T>0$. Let condition (i1) be satisfied and suppose that $F: \mathcal{R}_{+} \times X \rightarrow \mathcal{P}_{f c}(X)$ is measurable in its first variable and upper semicontinuous in its second. Assume that either (a) $F$ is bounded and $W(t, s)$ is compact for $t>s$, or (b) $F$ is compact. Assume that for every $M>0$ and every $\epsilon>0$ there exists an $L^{1}\left(\mathcal{R}^{+}, X\right)$-function $b=b(M, \epsilon)$, an integrably bounded function $H_{M}: \mathcal{R}_{+} \rightarrow \mathcal{P}_{w k c}(X)$ and $T=T(M, \epsilon)>0$ such that

$$
F(t, x) \subset H_{M}(t), \text { for almost all } t \in \mathcal{R}_{+},
$$


and

$$
F(t, x)-b(t) \subset \epsilon B_{1}(0), \text { for almost all } t \geq T \text { and all } x \in B_{M}(0) .
$$

Moreover, there exist constants $\alpha, N>0$ such that

$$
\|W(t, s)\| \leq N \exp \{-\alpha(t-s)\}, \text { for all } t, s \in R_{+} \text {with } t \geq s .
$$

Let $a \in X$ be a fixed vector. Then the operator $V$ defined by

$$
\begin{aligned}
V x \equiv\left\{y \in C_{b}: y(t)\right. & =W(t, 0) \widetilde{U}^{-1}[a-U \phi(\cdot, f)] \\
+ & \left.\int_{0}^{t} W(t, s) f(s) d s, t \in \mathcal{R}_{+}, \text {for some } f \in S_{F(\cdot, x(\cdot))}^{1}(\infty)\right\}
\end{aligned}
$$

is upper semicontinuous on bounded subsets of $C_{b}$ and maps bounded subsets of $C_{b}$ onto relatively compact subsets of $C_{b}$. If, in addition, all possible solutions $x_{\lambda} \in C_{b}$ of the inclusion $x-\lambda V x \ni 0, \lambda \in(0,1)$, are uniformly bounded, then problem (B) has a mild solution.

Proof. We show first that $V$ maps bounded subsets of of $C_{b}$ onto relatively compact subsets of the same space. We intend to prove first that the set

$$
K_{t}=\left\{\int_{0}^{t} W(t, s) f(s) d s: f \in S_{F(\cdot, x(\cdot))}^{1}(\infty) \text {, for some } x \in B_{M}^{C}(0)\right\}
$$

is relatively compact in $X$ for every $t \in \mathcal{R}_{+}$. This is obvious for $t=0$. We assume that $t>0$ and that assumption (a) holds. Then our conclusion follows as in the proof of Lemma 2.2 of Ward [17]. Let assumption (b) hold, let $x \in B_{M}^{C}(0)$ and let $f \in S_{F(\cdot, x(\cdot))}^{1}(\infty)$. Since $\|x(t)\| \leq M$, there exists a compact, convex set $Q \subset X$ such that

$$
f(s) \in F(s, x(s)) \subset Q, \text { for almost all } s \in[0, t] .
$$

We claim that the set

$$
B_{t} \equiv W(t,[0, t]) Q=\{W(t, s) x: s \in[0, t], x \in Q\}
$$

is compact. To see this, we let $\left\{y_{n}\right\} \subset B_{t}$. Then there exist sequences $\left\{s_{n}\right\} \subset$ $[0, t],\left\{x_{n}\right\} \subset Q$ such that $y_{n}=W\left(t, s_{n}\right) x_{n}, n \in \mathcal{N}$. We may assume that $s_{n} \rightarrow$ $s_{0} \in[0, t]$ and $x_{n} \rightarrow x_{0} \in Q$ as $n \rightarrow \infty$. Let $y_{0}=W\left(t, s_{0}\right) x_{0}$. Then

$$
\begin{aligned}
\left\|y_{n}-y_{0}\right\| & =\left\|W\left(t, s_{n}\right) x_{n}-W\left(t, s_{0}\right) x_{0}\right\| \\
& \leq\left\|W\left(t, s_{n}\right) x_{n}-W\left(t, s_{n}\right) x_{0}\right\|+\left\|W\left(t, s_{n}\right) x_{0}-W\left(t, s_{0}\right) x_{0}\right\| \\
& \leq\left\|W\left(t, s_{n}\right)\right\|\left\|x_{n}-x_{0}\right\|+\left\|W\left(t, s_{n}\right) x_{0}-W\left(t, s_{0}\right) x_{0}\right\| \\
& \leq N\left\|x_{n}-x_{0}\right\|+\left\|W\left(t, s_{n}\right) x_{0}-W\left(t, s_{0}\right) x_{0}\right\| \rightarrow 0 \text { as } n \rightarrow \infty .
\end{aligned}
$$

Now we apply the Mean Value Theorem (cf. Aubin and Cellina [2, p. 21]) in order to get

$$
\int_{0}^{t} W(t, s) f(s) d s \in t \overline{\mathrm{co}} B_{t} .
$$

This implies that $K_{t} \subset t \overline{\mathrm{co}} B_{t}$ and proves the relative compactness of the set $K_{t}, t \in$ $\mathcal{R}_{+}$.

We now show the stability of the set $D \equiv V B_{M}^{C}(0)$, i.e., we show that for every $\epsilon>0$ there exists $T=T(\epsilon)>0$ and $\delta=\delta(\epsilon)>0$ such that: if $u \in V x$ and 
$v \in V y$ are such that $\|u(T)-v(T)\|<\delta$, then $\|u(t)-v(t)\| \leq \epsilon$ for every $t \geq T$. We follow, partly, the proof of Theorem 2 of Przeradzki [16]. Fix $\epsilon>0$ and choose $T_{1}=T_{1}(\epsilon)>0$ such that

$$
F(t, x)-b(t) \subset[\epsilon \alpha /(8 N)] B_{1}(0) \text {, for almost all } t>T_{1} \text { and all } x \in B_{M}(0) .
$$

Assume that the bounded set $Q$ is such that $F(t, x) \subset Q$, for almost all $t \in\left[0, T_{1}\right]$ and all $x \in B_{M}(0)$, and let $\widetilde{M}$ be an upper bound for $Q$. Choose $T=T(\epsilon) \geq T_{1}$ so large that for $t \geq T$ and $s \leq T_{1}$ we have

$$
\|W(t, s)\| \leq N \exp \left\{-\alpha\left(T-T_{1}\right)\right\}<\frac{\epsilon}{8 \widetilde{M} T_{1}} .
$$

Letting $u \in V x, v \in V y$, for some $x, y \in B_{M}^{C}(0)$, we see that there exist $f \in$ $S_{F(\cdot, x(\cdot))}^{1}(\infty), g \in S_{F(\cdot, y(\cdot))}^{1}(\infty)$ such that

$$
u(t)=W(t, 0) \widetilde{U}^{-1}[a-U \phi(\cdot, f)]+\int_{0}^{t} W(t, s) f(s) d s, t \in \mathcal{R}_{+},
$$

and

$$
v(t)=W(t, 0) \widetilde{U}^{-1}[a-U \phi(\cdot, g)]+\int_{0}^{t} W(t, s) g(s) d s, t \in \mathcal{R}_{+} .
$$

Consequently, for $t \geq T$,

$$
\begin{aligned}
\| \int_{0}^{t} W(t, s)[ & f(s)-g(s)] d s\|\leq\| \int_{[0, t] \cap\left\{s>T_{1}\right\}} W(t, s)[f(s)-g(s)] d s \| \\
& +\left\|\int_{[0, t] \cap\left\{s \leq T_{1}\right\}} W(t, s)[f(s)-g(s)] d s\right\| \\
\leq & \left\|\int_{[0, t] \cap\left\{s>T_{1}\right\}} W(t, s)[f(s)-b(s)] d s\right\| \\
& +\left\|\int_{[0, t] \cap\left\{s>T_{1}\right\}} W(t, s)[g(s)-b(s)] d s\right\| \\
& +\left\|\int_{[0, t] \cap\left\{s \leq T_{1}\right\}} W(t, s)[f(s)-g(s)] d s\right\| \\
\leq & 2 \int_{[0, t] \cap\left\{s>T_{1}\right\}}\|W(t, s)\| \sup _{\|x\| \leq M}|F(s, x)-b(s)| d s \\
& +2 \int_{[0, t] \cap\left\{s \leq T_{1}\right\}}\|W(t, s)\| \widetilde{M} d s \\
\leq & \frac{\epsilon \alpha}{4 N} \int_{0}^{t} N \exp \{-\alpha(t-s)\} d s+\frac{2 \widetilde{M} \epsilon T_{1}}{8 \widetilde{M} T_{1}}=\frac{\epsilon}{2} .
\end{aligned}
$$

We can now use the fact that $H_{M}(t), t \in \mathcal{R}_{+}$, is integrably bounded and the fact that

$$
\|W(t, 0)\| \rightarrow 0, \text { as } t \rightarrow \infty,
$$

to show that we may choose $T \geq T_{1}$ so large that

$$
\|u(t)-v(t)\| \leq \epsilon, \text { for all } t \geq T .
$$


This finishes the proof of the stability of the set $D$. In the proof of the stability of the set $D$, we showed the stability of the set

$$
\begin{array}{r}
M_{2} \equiv\left\{u \in C_{b}: u(t)=\int_{0}^{t} W(t, s) f(s) d s, \text { for some } f \in S_{F(\cdot, x(\cdot))}^{1}(\infty)\right. \\
\text { and some } \left.x \in B_{M}^{C}(0)\right\} .
\end{array}
$$

Since the set $K_{t}$ is relatively compact in $X$ and $M_{2}$ is equicontinuous by virtue of Lemma 2 , we have the relative compactness of the set $M_{2}$. Since the mapping $u \rightarrow$ $W(\cdot, 0) \widetilde{U}^{-1} U u$ is a bounded linear operator on $C_{b}$ and $M_{2}$ is relatively compact, it follows that the set

$$
\begin{array}{r}
M_{3} \equiv\left\{u \in C_{b}: u(t)=W(t, 0) \widetilde{U}^{-1}\left(a-U \phi(\cdot, f) \text { for some } f \in S_{F(\cdot, x(\cdot))}^{1}(\infty)\right.\right. \\
\text { and some } \left.x \in B_{M}^{C}(0)\right\}
\end{array}
$$

is also relatively compact. Since $D \subset M_{2}+M_{3} \subset \overline{M_{2}}+\overline{M_{3}}$ and $\overline{M_{1}}, \overline{M_{2}}$ are compact, we have the relative compactness of the set $D$. Since $\overline{V B_{M}^{C}(0)}$ is compact, $V$ will be upper semicontinuous, as a mapping from $B_{M}^{C}(0)$ to $\overline{V B_{M}^{C}(0)}$, if its graph is closed in $B_{M}^{C}(0) \times \overline{V B_{M}^{C}(0)}$ (cf. [2, p. 42]). Thus, it suffices to show that its graph is closed in $B_{M}^{C}(0) \times C([0, T], X)$. To show this, we let $x_{n} \in B_{M}^{C}(0), y_{n} \in V x_{n}$ be such that $\left(x_{n}, y_{n}\right) \rightarrow(x, y)$ uniformly on $\mathcal{R}_{+}$. Then there exist functions $f_{n} \in S_{F\left(\cdot, x_{n}(\cdot)\right)}^{1}(T)$ such that

$$
y_{n}(t)=W(t, 0) \widetilde{U}^{-1}\left[a-U \phi\left(\cdot, f_{n}\right)\right]+\int_{0}^{t} W(t, s) f_{n}(s) d s, t \in[0, T] .
$$

Since $\mathcal{S}_{H_{M}}^{1}(\infty)$ is weakly compact in $L^{1}\left(\mathcal{R}^{+}, X\right)$, by Lemma 1 , we have that, passing to a subsequence if necessary, $f_{n} \rightarrow f \in \mathcal{S}_{H_{M}}^{1}(\infty)$ weakly in $L^{1}\left(\mathcal{R}^{+}, X\right)$. Invoking Mazur's theorem, there exists a sequence of terms $z_{n} \in \operatorname{co}\left(\bigcup_{k \geq n} f_{k}\right)$ such that $z_{n} \rightarrow f$ strongly in $L^{1}\left(\mathcal{R}^{+}, X\right)$. We may assume that $z_{n}(t) \rightarrow f(t)$ strongly as $n \rightarrow \infty$, for $t \in \mathcal{R}_{+} \backslash \mathcal{N}$, where $\mathcal{N}$ is a set of measure zero. We fix $t \in \mathcal{R}_{+} \backslash \mathcal{N}$. Since $F$ is upper semicontinuous in its second variable, we know that given a ball $B_{r}(0)$, for some $r>0$, there exists $n \geq 1$ such that, for $k \geq n$, we have

$$
F\left(t, x_{k}(t)\right) \subset F(t, x(t))+B_{r}(0),
$$

which implies

$$
\overline{\mathrm{co}}\left(\bigcup_{k \geq n} F\left(t, x_{k}(t)\right)\right) \subset F(t, x(t))+\overline{B_{r}(0)}
$$

i.e.,

$$
f(t) \in F(t, x(t))+\overline{B_{r}(0)} .
$$

Since $r>0$ is arbitrary, $f(t) \in F(t, x(t))$ for every $t \in \mathcal{R}_{+} \backslash \mathcal{N}$. Thus, $f \in$ $\mathcal{S}_{F(\cdot, x(\cdot))}^{1}(\infty)$. It is easy to see, by using Lebesgue's Dominated Convergence Theorem, that

$$
y(t)=W(t, 0) x_{0}+\int_{0}^{t} W(t, s) f(s) d s, t \in \mathcal{R}_{+} .
$$

This completes the proof of the first part of the theorem. 
If the assumption on the solvability of $x-\lambda V x \ni 0$, in the second part of the theorem, is made, then for every solution $x_{\lambda}$ of this inclusion we have $\left\|x_{\lambda}\right\| \leq \widetilde{M}$, where $\widetilde{M}$ is a fixed positive constant. In order to obtain a mild solution to problem (B) by Ma's Theorem 16.1 in [12], it suffices to restrict the mapping $V$ to the ball $B_{M}^{C}(0)$, where $M$ is any constant in $(\widetilde{M}, \infty)$.

A method for creating concrete examples of linear and nonlinear compact evolution operators, generated by time-dependent $m$-accretive operators, has been developed by Kartsatos in [10].

For local existence results involving functional perturbations of nonlinear $\mathrm{m}$ accretive operators, generating compact evolution operators, the reader is referred to Kartsatos and Shin [11]. It would be interesting to see extensions of our results to the existence of extremal solutions of boundary value problems as in [15].

\section{REFERENCES}

[1] G. Anichini, Nonlinear problems for systems of differential equations, Nonlinear Anal. TMA 6 (1977), 691-699. MR 58:28782

[2] J. P. Aubin and A. Cellina, Differential Inclusions, Springer-Verlag, New York, 1984. MR $\mathbf{8 5 j}: 49010$

[3] A. Friedman, Partial Differential Equations, Holt, Rinehart and Winston, New York, 1969. MR 56:3433

[4] C. J. Himmelberg, Measurable selections, Fund. Math. 87 (1975), 53-72. MR 51:3384

[5] S. Hu and N. S. Papageorgiou, On the existence of periodic solutions for a class of nonlinear evolution inclusions, Boll. Un. Mat. Ital. B 7 (1993), 591-605. MR 94k:34120

[6] A. G. Kartsatos, The Leray-Schauder theorem and the existence of solutions to boundary value problems on infinite intervals, Indiana Univ. Math. J. 23 (1974), 1021-1029. MR 49:5448

[7] A. G. Kartsatos, Nonzero solutions to boundary value problems for nonlinear systems, Pacific J. Math. 53 (1974), 425-433. MR 51:13337

[8] A. G. Kartsatos, Locally invertible operators and existence problems in differential systems, Tôhoku Math. J. 28 (1976), 167-176. MR 55:3390

[9] A. G. Kartsatos, Boundary value problems for abstract evolution equations, Nonlinear Anal. TMA 3 (1978), 1-8. MR 80i:34104

[10] A. G. Kartsatos, A compact evolution operator generated by a nonlinear time-dependent $m$-accretive operator in a Banach space, Math. Ann. 302 (1995), 473-487. MR 93c:47104

[11] A. G. Kartsatos and K. Y. Shin, Solvability of functional evolutions via compactness methods in general Banach spaces, Nonlinear Anal. TMA 21 (1993), 517-535. MR 94h:34100

[12] T. W. Ma, Topological degrees of set-valued compact fields in locally convex spaces, Dissert. Math. 92 (1972), 1-43. MR 46:8214

[13] N. S. Papageorgiou, A stability result for differential inclusions in Banach spaces, J. Math. Anal. Appl. 118 (1986), 232-246

[14] N. S. Papageorgiou, Boundary value problems for evolution inclusions, Comment. Math. Univ. Carolinae 29 (1988), 355-363. MR 89k:34018

[15] N. S. Papageorgiou, Boundary value problems and periodic solutions for semilinear evolution inclusions, Comment. Math. Univ. Carolinae 35 (1994), 325-336. CMP 94:15

[16] B. Przeradzki, The existence of bounded solutions for differential equations in Hilbert spaces, Ann. Polon. Math. 56 (1992), 103-121. MR 93d:34109

[17] J. R. Ward, Boundary value problems for differential equations in Banach spaces, J. Math. Anal. Appl. 70 (1979), 589-598. MR 80i:34110

[18] P. Zecca and P. Zezza, Nonlinear boundary value problems in Banach spaces for multivalued differential equations on a non-compact interval, Nonlinear Anal. TMA 3 (1979), 347-352. MR 80h:34084

Department of Mathematics, University of South Florida, Tampa, Florida 33620-5700

E-mail address: ding@chuma.usf.edu, hermes@gauss.math.usf.edu 